\title{
New trends in computer math teaching
}

\author{
Agustin de la Villa1, Alejandro Lois², Liliana Milevicich², Angel Martin del Rey³ and Gerardo \\ Rodriguez ${ }^{3}$ \\ ${ }^{1}$ Applied Mathematics, Pontificia Comillas University, Madrid, Spain \\ 2. Applied Mathematics, National Technological University, Buenos Aires, Argentina \\ ${ }^{3}$ Applied Mathematics, Salamanca University, Salamanca, Spain \\ For correspondence: avilla@upcomillas.es
}

\begin{abstract}
In the first section of this paper we analyze the possibilities for using the new technologies and the corresponding changes in the methodologies to be used.We continue with some thoughts on the use of Computer Algebra Systems (CAS) in cases of applications of mathematics in Engineering. The use of CAS is now a common practice that has changed the behavior of teachers and students in teaching. Therefore, we must design courses considering the great potential of computer algebra systems to enhance learning.The methodological change should not be exclusive to the teaching methodology. At present, there is a debate on how to integrate CAS to help in assessment tasks and how to use CAS without limiting its use to the practical exams in Mathematics Laboratories.Thanks to the possibilities provided by the use of computers, it seems reasonable to change the teaching materials and the way in we assess the students.Finally, we will describe two experiences for an integrated use of a CAS in basic math courses that is currently being offered in Spanish engineering schools.
\end{abstract}

Keywords: Computer Algebra Systems, Assessments, e-learning.

\section{Introduction}

In this introduction some ideas about the new European scenario: European Higher Education Area (EHEA) and different possibilities for using the Computer in teaching will be exposed.

The current educational scenario with regards to the teaching of mathematics in engineering schools is a completely different scenario from the one of the last decade of the twentieth century. This rapid change has been produced for two main reasons:

(i) the regulatory changes in the structure and contents of the various engineering degrees derived largely from the launch of the European Higher Education and

(ii) (ii) the technology changes that have allowed the current teaching revolution.

The European Higher Education Area (in http://www.ehea.info/ accessed March 2014) has adopted a new model of learning and teaching based on competences, opposite to the previous system based on the contents. This model must modify substantially the teaching methodology used in the last decades.

The new technologies have changed teaching methodologies in the past twenty years. The use of computers is now a common practice that has changed the behavior of teachers and students. General ideas about their use can be shown in Artigue (2002), Drijvers, Trouche (2010), Lagrange et al. (2003), Limniou, Smith (2012), Lois et al. (2011), Lois et al. (2013) and Lois et al. (2013).

This change has been carried out, to a greater or lesser extent, in all Engineering subjects, including Mathematics.

From the concept of e-learning, an emerging concept built up ten years ago, we are moving to the concepts of blended learning or the new modern concept of u-learning. Multiple learning opportunities and possibilities have been open. 
To address the challenges created nowadays, the different higher education institutions have substantially modified their teaching methodologies compared to the methodolgies used in the late 80 's. ICTs have greatly contributed to this new situation. From theoretical and practical lectures, using the traditional method of chalk and board, we have transitioned to a Learning Management System (LMS), usually based on MOODLE, where teaching materials are uploaded and can be used from anywhere and in any electronic format ( $\mathrm{u}$ - learning). New challenges are posed and a collaborative work is necessary. Therefore, the use of ICT requires the development of new teaching materials to be used by the students in very different circumstances (individual work and team work mainly). The solution of projects or selected problems proposed to the students also requires both self-learning and teamwork to allow them to acquire the skills and competencies that the curriculum requires.

The previous tasks (elaboration of worthy materials, search projects, etc.) cannot be done individually and, therefore, the formation of large teaching teams involved in a collaborative work will be imperative for addressing this challenge.

In this sense, in the last ten years, numerous teaching teams have been created and developed, not limited to the scope of a university, with the objective of continuing with the development of educational materials and designing didactic strategies to renew the teaching methodology of mathematics. There have been many experiences of transnational level through European projects supported by the Leonardo da Vinci program and through agreements signed between universities in different countries. The authors of this article have participated in some of them, through European projects: dMath (in Bringslid et al. (2007), EVLM (see figure 1 and http://portalevlm.usal.es/ accessed March 2014), WEBMLS (in https://myweps.com/moodle/ accessed March 2014). The final product of these projects has been an educational portal for general purposes. Materials developed in these projects have been developed in the different languages of the countries participating in them ensuring a wide dissemination and use thereof.
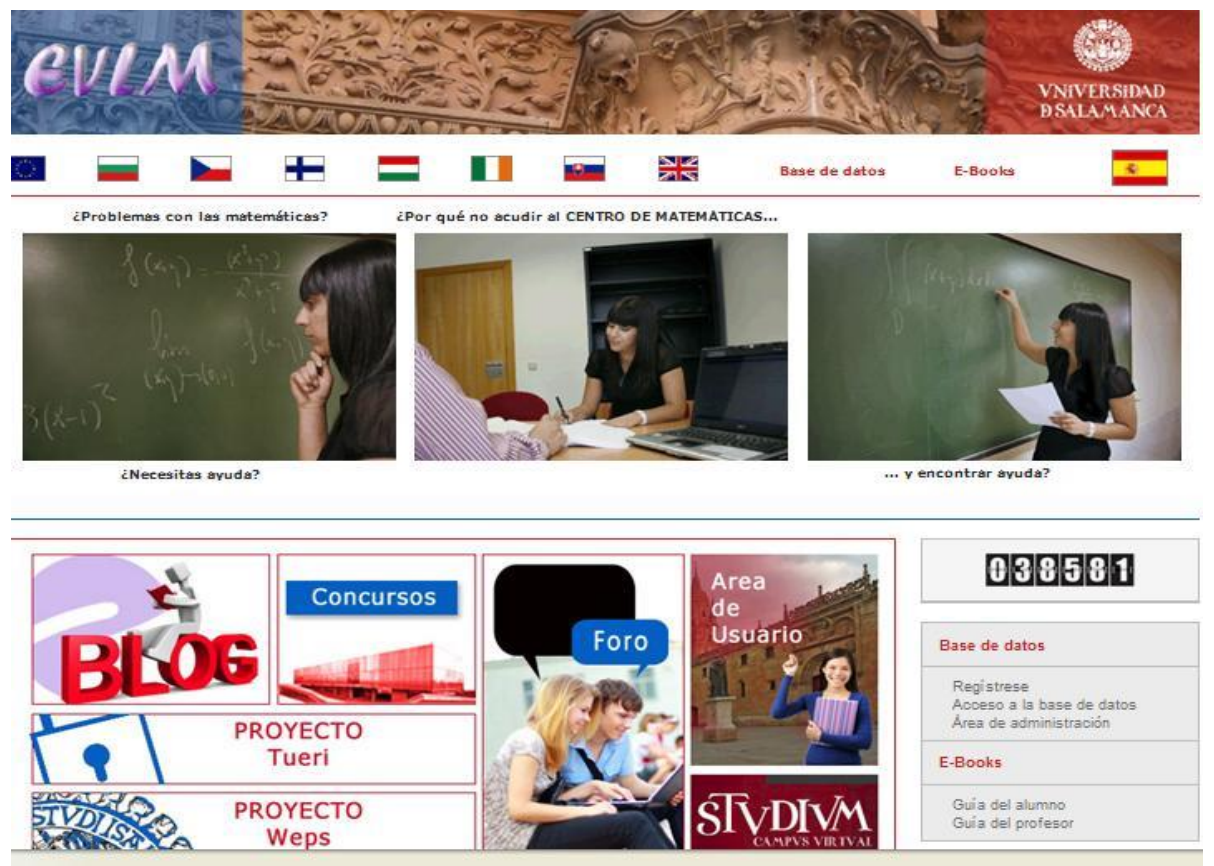

Figure 1. Spanish EVLM portal.

The authors of this article are currently developing materials for a course in Calculus in Several Variables. These materials are fruit of the collaboration between teachers of the University of Salamanca and Universidad Pontificia Comillas in Spain and the National Technological University of Argentina in Buenos Aires.

Although it is a subject of ongoing debate and we are still far from a broad consensus in this sense, the use of ICT should be extended to develop new assessment protocols. The educational paradigm 
shift towards teaching focused on the acquisition of competencies by the students must be extended to a change in assessment methods. The traditional math exams, where students respond to different questions with pencil and paper, must be updated. The use of all technological possibilities at our disposal allows the realization of different tests that allow us to assess the competencies that the new educational model claims.

Different LMS allow the implementation of automatic questionnaires using different systems such as STACK and WIRIS (figures 2 and 3). These questionnaires can be used not only as a self-assessment test where the student receives immediate feedback on his progress, but also can be used as part of the assessment process. LMS allow different forms of questionnaires that should help to establish a new assessment protocol that replaces the traditional math exam.

In short, the educational possibilities that ICTs allow have substantially altered the teaching work, showing, moreover, that the answer to the new challenges in the teaching-learning process has to be the outcome of a very collaborative work between all the elements in the process.

\section{The CAS: Methodological changes and assessments.}

The different CAS have also contributed to changing the teaching methodology. The possibilities offered by the developers of the CAS added in the continuous updates have facilitated their use by both teachers and students. We can consider, therefore, different stages in the use of CAS in mathematics` teaching.

The use of different CAS in the teaching process has increased as a consequence of the ease of use and the improved capabilities and the versatility. In the first stage, the use of computers was limited, outside of the traditional classroom, to being used as a fundamental tool in Numerical Calculus courses. To use the various programs, users had to learn to handle the software and time has to be spent teaching them the programming languages used in the numerical algorithms.

The widespread use of computers in the universities and the emergence of the first CAS increased the use of computers as a support in the teaching process through the creation of Mathematical Laboratories in the Schools of Engineering (at least in Spain) during the last decade of the twentieth century. The different software packages were used to develop different practical sessions that complement the teaching in the traditional classroom. The choice of CAS to be used was still important then, because it was necessary to devote time to training students in the use of the chosen CAS.

The situation has drastically changed in the past years due the ease of use of the updated versions of the CAS. Some capabilities of the CAS: graphical, symbolic and numerical capacities, interaction and simulation, etc., allow us to redesign teaching and learning for many mathematical concepts. The main idea about the CAS is the following: the CAS used is not important, we should, instead, pay attention to the way the CAS is used. We need to encourage our students to do mathematics with a CAS but they don't need to be experts in the CAS.

Nowadays we can find numerous experiments carried out to check that the use of the CAS (in Franco et al. (2000), Alonso et al. (2001), García et al. (2006) and García et al. (2010)). Also the comparison for using different CAS has been analyzed and we can conclude the CAS used is not important because it could repeat the experience with another CAS with similar characteristics ( see García et al. (2011)). In the last years it has been possible to find experiences of integrated use of the CAS in Engineering Mathematical courses (in García et al. (2005)).

The power and versatility of the current CAS allow a further step in their use. We can promote the development of toolboxes that students can use in many different ways. Toolboxes must be understood as the implementation of automatic procedures for using the CAS to solve real or technical problems related with the mathematical topics analyzed in the corresponding subject. The toolboxes can be used by the students in the mathematical subjects and technical subjects during their 
studies or in their professional live. The toolboxes can begin with some procedures provided by the teacher of the particular subject, but can be extended and shared by students in another example of collaborative work which, in general, is a soft skill that students must acquire mathematics subjects (in García et al. (2009)).

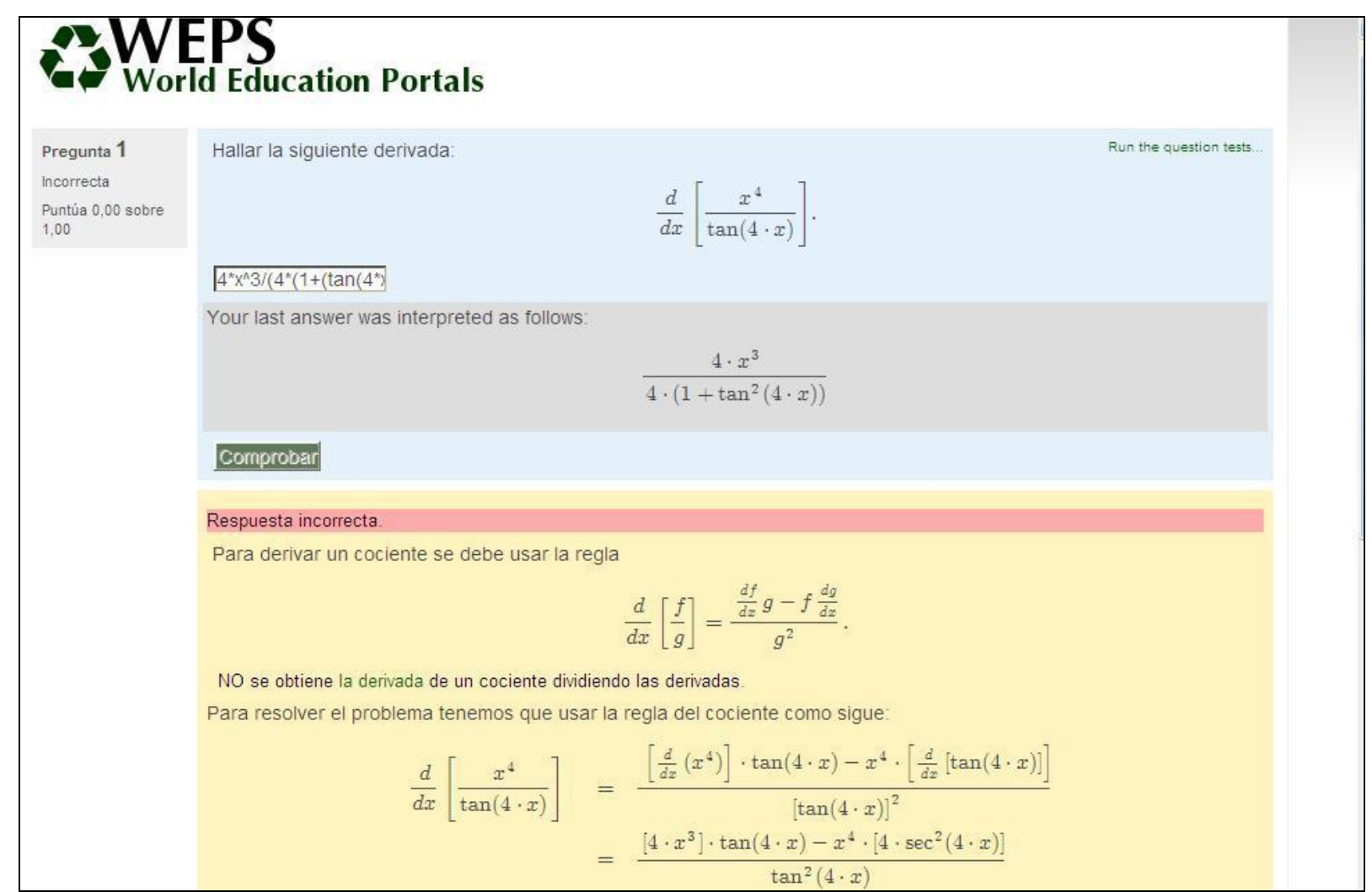

Figure 2. An example of STACK questionnaire (in Spanish).



Figure 3. An example of WIRIS questionnaire (in Spanish).

The methodological change does not only appear in teaching practice. At present, there is a debate on how to integrate CAS to help in assessment tasks and how to use CAS without limiting its use to the practical exams in Mathematics Laboratories. In this sense, it is necessary to incorporate the use of 
CAS into the new assessment protocol mentioned above, so that the CAS is present throughout the entire teaching and assessment process. For more information see García et al. (2012) and García et al. (2014).

\section{The materials to be used and the methodology for the teaching.}

It seems reasonable to change the teaching materials and teaching practice but this change has to be reflected in the assessment tasks, integrating it into teaching and the learning process. Therefore, we can offer our students traditional materials such as books written by ourselves (see García et al. (2007)), CAS files, etc.

It is necessary to develop materials that can be used in different teaching scenarios: face to face teaching, distance learning, etc. This is a collaborative task that cannot approach from an individual perspective. Also the educational materials must be taken into account for their many contexts. Therefore, by our previous experience in the projects related before, the development of teaching materials should follow a modular structure.

Following the modular structure from a big database, containing documents with theoretical lectures, solved and proposed problems, and assessment materials in very different supports (videos, presentations (slides shows) (see figure 4), pdf files, files with different use of CAS, etc.), would be created.

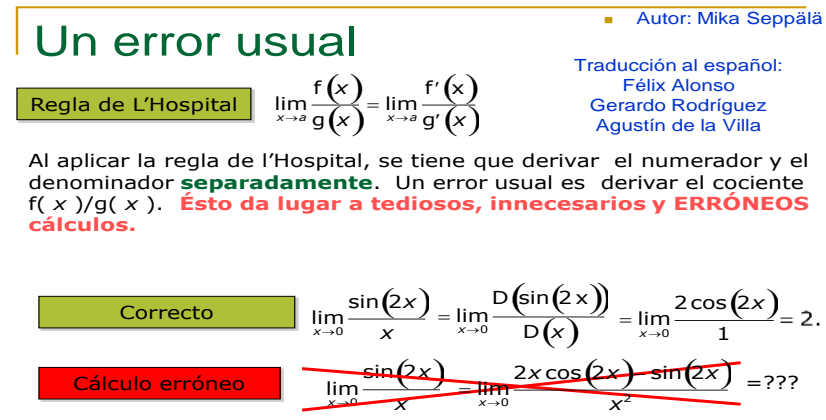

No caer en este error. El cálculo es incorrecto!!!!

Derivación. Aplicaciones de la derivada. regla de L'Hospital

Figure 4. An example of slide about L'Hôpital rule in Spanish.

For the different contents and different files, according the required level of deep can be prepared. These files, called Reusable Learning Objects (RLO), are the basic skeleton that the teacher uses, in various ways, according to the specific educational scenario and the characteristics of the students following the course. The materials can be assembled for activities including individual learning, group activities and recovery activities that students may need. For a particular course we can offer our students traditional materials, books written by ourselves, CAS files and RLO taken from a database. Also the materials needed in introductory courses for students follow a particular course with deficiencies in their initial training can be produced.

The use of Learning Content Management Systems (LCMS), mainly MOODLE, must produce a good mix of learning and assessment activities.

In this way the initial objective of the teacher should be to achieve a homogeneous starting point for the corresponding course. That is, the processed materials can be used in a personalized way based on the characteristics of each student. This action clearly improves the personalized tutorial action according to the characteristics of each student. Again ICT plays an essential role in this model. 


\section{The experiences.}

We will describe two experiences, carried out in the University of Salamanca and the Polytechnic University in Madrid. In these experiences we proposed the use of a CAS integrated as much as possible in basic math courses currently offered in Spanish engineering schools. The experiences are the result of many years working in this area. We will describe these experiences briefly.

\section{Salamanca University experience:}

The topic Applied Mathematics I, corresponding to the Technical Architecture Degree in the Polytechnic School of Zamora has a standard course content Calculus One Variable, with special emphasis on the differential and integral calculus. All students have access to Mathematica 9.0, which is currently available in the campus license version in the University of Salamanca. For this reason, the material used in the course is based on this CAS. During the first week of the course the most important features of Mathematica are explained. Using the materials made available to students in the project portal EVLM (http://portalevlm.usal.es/) and the assistance provided by the Mathematica package, the students are quickly CAS users. They do not have any trouble using Mathematica like integrated material in the course.

Mathematica is used in many different ways, not only for practical sessions carried out in the computer rooms. When the student has assimilated the basic routines and the basic concepts of a particular subject, they usually use the CAS to perform the calculations associated with the team work completed in the subject. Thus, the characteristics of CAS allow us to advance quickly on issues such as the representation of functions, the calculation of derivatives and integrals. Traditionally, for example, at least in Spain, much of the time of the Calculus I course was devoted to show different techniques for calculating primitive functions "by hand", while the power of the CAS allows us currently to avoid this calculations with paper and pencil and tackle new challenges in the field of mathematical modeling. The same strategy can be applied to the calculation of partial derivatives.

The assessment of this course is done as follows:

- $60 \%$ of the final grade is acquired from the average obtained in the performance of individual or team group works. This list of works include written assignments, participation in the matters raised in the course blog (http://portalevlm.usal.es/blogs/nuevo_aplicada/) and practical sessions carried out with Mathematica in the Computer Room.

- $40 \%$ of the final grade is obtained from the average performance of four individual tests or exams (two traditional tests, one on-line questionnaire and another exam with Mathematica) throughout the semester.

The academic results are very satisfactory. In the first call of the current academic year 2013-14, 85\% of students passed the subject. We believe that the experience must advance and consolidate. Taken into account the new forms and possibilities of assessments we have to modify the assessment system, avoiding the excessive weight that the traditional written examination has in the final grade.

\section{Polytechnic University experience:}

The Linear Algebra course in the Mechanical Engineering Degree in Polytechnic University of Madrid has a standard content: Vector spaces, linear transformations, Jordan canonical form, Euclidean spaces and orthogonal transformations. In this course, the CAS MAXIMA has been fully integrated into the teaching experience. Its use in the lectures is typical when the teacher deems it appropriate. It can also be used by the students anywhere since the software is free and most of the students have a laptop. The assessment of this course is done as follows:

- $60 \%$ of the final grade is obtained through three written exams, in which the use of CAS is allowed. The weighting of the exams is $15 \%, 30 \%$ and $35 \%$ respectively because the contents of the exam are cumulative.

- $40 \%$ of the final grade is obtained using MAXIMA: $10 \%$ can be obtained by solving a collection of problems in team work. The other $10 \%$ is obtained through one exam using MAXIMA. 
The academic results are relatively satisfactory. Approximately $70 \%$ of the students who have taken the course have passed the subject, a very high percentage for the subjects of mathematics in engineering studies in Spain.

\section{Conclusions and further work.}

Taking into account the above paragraphs we can conclude:

It is necessary to adapt the teaching methodology and assessment methods to modern times, adapting to teaching strategies that are very different from those that were in place a few years ago.

In math we use the computer to introduce, in the most integrated way possible, CAS in different disciplines.

The new methodologies and the use of LCMS (learning content management systems) should allow teaching "a la carte" and almost personalized.

The production of teaching materials, due to their complexity, should be addressed by multidisciplinary teams from several universities. An editorial committee should be in charge of the organization, style, contents, etc. of these materials. The experiences carried out are extremely positive taking judging by the academic results.

\section{References}

Alonso, F., Garcia, A. Garcia, F, Hoya, S., Rodriguez, G., Villa, A. (2001). Some unexpected results using computer algebra systems. International Journal of Computer Algebra in Mathematics Education. Vol 8 number 3. pp 239-252.

Artigue, M. (2002). Learning Mathematics in a CAS Environment: The Genesis of a Reflection about Instrumentation and the Dialectics between Technical and Conceptual work. International Journal of Computers for Mathematical Learning, 7, 245-274

Bringslid, O. Rodríguez, G. Villa, A. de la (2007) Dmath: A European project for the restatement of engineering mathematics teaching. European Journal of Engineering Education. Vol. 32.1. pp. 1-12

Drijvers, P., Trouche, L. (2010). Handheld technology for mathematics education: flashback into the future. ZDM Mathematics Education 42, 667-681

Franco, A., García, A., García, F., Gonzalez, F.J., Hoya, S., Rodriguez, G., Villa, A. de la (2000). Learning Calculus of Several Variables with New Technologies. International Journal of Computer Algebra in Mathematics Education, 2000. Vol 7 number 4. pp. 295-309.

García, A. García, F, Rodriguez, G., Villa, A. (2006). Some Strategies in the use of CAS in the teaching of Mathematics at Engineering Schools: A Spanish Perspective. Proceedings of 13th SEFI Maths Working Group Seminar. Kongsberg (Norway).

García, A. García, F, Rodriguez, V., Rodriguez, G., Villa, A. (2010). “One variable Calculus: A Spanish overview in accordance with EHEA". Proceedings 15th SEFI MWG Seminar and 8th Workshop GFC. Wismar (Germany)

García, A., García, F., Rodríguez, G, de la Villa, A. (2011). Could it be possible to replace DERIVE with MAXIMA? The International Journal for Technology in Mathematics Education. Vol. 18-3 pp. 137-142. ISSN: 1744-2710

García, A., García, F., Rodríguez, G, de la Villa, A. (2005). Una propuesta de innovación educativa: Una enseñanza integrada del cálculo infinitesimal en las escuelas de ingeniería. Proceedings del XIII Congreso de Innovación Educativa en las Enseñanzas Técnicas. Maspalomas (Gran Canaria)

García, A., García, F., Rodríguez, G, de la Villa, A. (2009)). A toolbox with DERIVE. The Derive Newsletter, 76, pp. 5-13.

García, A., García, F., Rodríguez, G, de la Villa, A (2012). Small projects: Learning and Assessing Competencies: New challenges for Mathematics in Engineering Degrees in Spain. Proceedings of 16th SEFI MWG Seminar. Salamanca (Spain)

García, A., García, F., Rodríguez, G, de la Villa, A. (2014). Changing Assessment Methods: New rules, new roles. Journal of Symbolic Computation., vol 61-62 pp 70-84.

García, A., García, F., Rodríguez, G, de la Villa, A.(2007). CALCULO I: Teoría y problemas de Análisis Matemático en una variable. Third edition. Editorial CLAGSA. Madrid. ISBN 978-84-921847-2-9

Lagrange, J.B., Artigue, M., Laborde, C., Trouche, L. (2003). Technology and mathematics education: multidimensional overview of recent research and innovation. In: F. K. S. Leung (Ed.), Second international handbook of mathematics education, Vol. 1 (pp. 237-270). Dordrecht: Kluwer Academic Publishers

Limniou, M., Smith, M. (2012). The role of feedback in e-assessments for engineering education. Education and Information Technologies, 17(4)

Lois, A., Milevicich, L., Rodríguez, G., de la Villa, A. (2011). Perspectiva de las TIC'S en la educación superior en Iberoamérica. P. Lestón (Ed), Acta Latinoamericana de Matemática Educativa 24, pp. 1170-1178. México: Comité Latinoamericano de Matemática Educativa.

Lois, A., Milevicich, L., Rodríguez, G., de la Villa, A. (2013). La revolución tecnológica en la enseñanza de las matemáticas: el nuevo paradigma ¿es una oportunidad de cambio o un simple engaño? R. Flores (Ed), Acta Latinoamericana de Matemática Educativa 26, pp. 1867-1876. México: Comité Latinoamericano de Matemática Educativa.

Lois, A., Milevicich, L., Rodríguez, G., de la Villa, A. (2013). Enseñar Matemática: un reto en el nuevo paradigma tecnológico. R. Flores (Ed), Acta Latinoamericana de Matemática Educativa 26, pp. 1859-1866. México: Comité Latinoamericano de Matemática Educativa. 